\title{
Molecular cloning, homology modeling and site-directed mutagenesis of vanadium- dependent bromoperoxidase (GcVBPO1) from Gracilaria changii (Rhodophyta)
}

\begin{abstract}
Vanadium-dependent haloperoxidases belong to a class of vanadium enzymes that may have potential industrial and pharmaceutical applications due to their high stability. In this study, the 5Niflanking genomic sequence and complete reading frame encoding vanadium-dependent bromoperoxidase (GcVBPO1) was cloned from the red seaweed, Fracilaria changii, and the recombinant protein was biochemically characterized. The deduced amino acid sequence of GcVBPO1 is 1818 nucleotides in length, sharing $49 \%$ identity with the vanadium-dependent bromoperoxidases from Corralina officinalis and Cor. pilulifera, respectively. The amino acid residues associated with the binding site of vanadate cofactor were found to be conserved. The Km value of recombinant GcVBPO1 for Brī was $4.69 \mathrm{mM}$, while its Vmax was 10.61 Ekat mgī 1 at pH 7. Substitution of Arg379 with His379 in the recombinant protein caused a lower affinity for $\mathrm{Brī}$, while substitution of Arg379 with Phe379 not only increased its affinity for Brī but also enabled the mutant enzyme to oxidize Clī . The mutant Arg379Phe was also found to have a lower affinity for II , as compared to the wild-type GcVBPO1 and mutant Arg379His. In addition, the Arg379Phe mutant has a slightly higher affinity for $\mathrm{H} 2 \mathrm{O} 2$ compared to the wild-type GcVBPO1. Multiple cis-acting regulatory elements associated with light response, hormone signaling, and meristem expression were detected at the $5 \mathrm{Aj}$ flanking genomic sequence of GcVBPO1. The transcript abundance of GcVBPO1 was relatively higher in seaweed samples treated with 50 parts per thousand (ppt) artificial seawater (ASW) compared to those treated in 10 and 30 ppt ASW, in support of its role in the abiotic stress response of seaweed.
\end{abstract}

Keyword: Gracilaria changii; Red seaweed; Site-mutagenesis; Gracilariales; Vanadiumdependent bromoperoxidase 